\title{
Toward the Theory of Stochastic Condensation in Clouds. Part I: A General Kinetic Equation
}

\author{
VITALY I. KHVOROSTYANOV \\ Department of Meteorology, University of Utah, Salt Lake City, Utah \\ Judith A. CURRY \\ Department of Aerospace Engineering Sciences, Program in Atmospheric and Oceanic Sciences, \\ University of Colorado, Boulder, Colorado
}

(Manuscript received 7 August 1997, in final form 11 February 1999)

\begin{abstract}
In order to understand the mechanisms of formation of broad size spectra of cloud droplets and to develop a basis for the parameterization of cloud microphysical and optical properties, the authors derive a general kinetic equation of stochastic condensation that is applicable for various relationships between the supersaturation relaxation time $\tau_{f}$ and the timescale of turbulence $\tau_{L}$. Supersaturation is considered as a nonconservative variable, and thus additional covariances and a turbulent diffusion coefficient tensor that is dependent on the supersaturation relaxation time, $k_{i j}\left(\tau_{f}\right)$, are introduced into the kinetic equation. This equation can be used in cloud models with explicit microphysics or can serve as a basis for development of parameterizations for bulk cloud models and general circulation models.
\end{abstract}

\section{Introduction}

The characteristics and evolution of the cloud droplet size spectra determines cloud radiative properties and the formation of precipitation. To account for these processes in cloud-resolving models and climate models requires correct understanding and then parameterization of cloud microstructure and its dependence on predicted atmospheric parameters.

Condensation theory for a constant updraft has been shown to narrow the droplet spectrum with time (e.g., Mordy 1959; Neiburger and Chien 1960; Buikov 1961). Since broad spectra are observed in clouds and are required to initiate collision and coalescence growth of precipitation, various hypotheses and approaches have been developed to explain and to describe broad spectra in clouds. Warner (1969), Baker and Latham (1979), and Telford and Chai (1980) have attempted to explain the broadening of the size spectra and occurrence of the bimodal spectra and large droplets (before coagulation) by mixing of cloud parcels with environmental air. Additional mechanisms that have been proposed include horizontal inhomogeneities in vertical velocity (e.g.,

Corresponding author address: Vitaly I. Khvorostyanov, Department of Meteorology, University of Utah, Salt Lake City, UT 84111 E-mail: vitalyk@atmos.met.utah.edu
Mazin 1968) and in cloud condensation nuclei (CCN) (Kabanov et al. 1970), microscopic supersaturation associated with random distribution of drops (Srivastava 1989), and sedimentation of the cloud droplets (Baker et al. 1984; Considine and Curry 1996).

The theory of stochastic condensation attributes the presence of broad drop size spectra in clouds to the occurrence of condensation in a turbulent medium. This theory was developed intensively during the period 1960-80. While considerable research was devoted to the explanation of the various mechanisms of the broadening of the droplet size spectra (see reviews in Cotton and Anthes 1989; Pruppacher and Klett 1997), the treatment that is mathematically most promising describes stochastic condensation in terms of kinetic equations that allow direct calculations of the evolution of the size spectra. These equations have been derived for two limiting cases: the low-frequency regime in which the characteristic Lagrangian timescale of turbulent fluctuations, $\tau_{L}$, is much greater than the supersaturation absorption time, $\tau_{f}$, that is, $\tau_{L} \gg \tau_{f}$; and the high-frequency regime in which $\tau_{L} \ll \tau_{f}$. Since $\tau_{f} \sim 1-10 \mathrm{~s}$ in a developed cloud, and $\tau_{L} \sim 5-10 \mathrm{~min}$, it was believed in earlier studies that the high-frequency approximation might be representative of rapidly changing conditions such as might occur in an entrainment zone or during the initial phase of condensation, while the low-frequency approximation might be representative of a developed 
cloud that is quasi-steady. More detailed analysis of the turbulence in clouds showed that there are pronounced maxima in the turbulent power spectra of vertical velocity at the frequences $\omega \sim 10^{-3}-10^{-2} \mathrm{~s}^{-1}$ (e.g., Curry et al. 1988; Sassen et al. 1989). The supersaturation relaxation (absorption) time in the cloud regions with smaller particles concentrations can be $\tau_{f} \sim 30-60 \mathrm{~s}$ for liquid clouds and can exceed $\tau_{f} \sim 30-180 \mathrm{~min}$ for crystalline clouds (e.g., Khvorostyanov and Sassen 1998). Thus the timescales $\tau_{f}$ and $\tau_{L}$ can be quite comparable and their relation can change during cloud evolution; hence more general kinetic equations for arbitrary relations between $\tau_{f}$ and $\tau_{L}$ are required.

The method of kinetic equations was introduced in cloud physics by Buikov (1961, 1963), who wrote the kinetic equation by analogy with the diffusion equation but incorporated an additional term describing condensational growth. When deriving analytical solutions to the kinetic equations, the additional covariances that appear due to turbulent fluctuations of supersaturation and the size distribution function were neglected. It will be shown below that this approach is equivalent to the high-frequency approximation, $\tau_{L} \ll \tau_{f}$. Another version of the kinetic equations that corresponds to the lowfrequency approximation was derived by Levin and Sedunov (1966) and Sedunov (1974), who developed the theory of stochastic condensation, which attributes the presence of broad drop size spectra in clouds to turbulent fluctuations. The stochastic method treats supersaturation and droplet growth rate as stochastic variables and replaces the usual operator of turbulent diffusion $K \partial / \partial x$ by the operator $K(\partial / \partial x+A \partial / \partial s)$ for a nonconservative substance $s$ (here, the droplet surface) where $A$ is proportional to the vertical velocity. The solution to this equation predicts the growth of the peak radius with time and dispersion of the drop size spectra that asymptotes with time toward a constant value.

More rigorous and detailed derivations of the equation of stochastic condensation using Reynolds averaging and more careful evaluation of covariances were performed by Stepanov (1975), Voloshchuk and Sedunov (1977), and Manton (1979) using the method of perturbation theory, which is valid for weak turbulence (fluctuations are smaller than the mean values). This derivation was generalized by Merkulovich and Stepanov (1977) to account for curvature and solution effects in droplet growth.

The kinetic equations derived in the aforementioned studies are valid in the low-frequency approximation, that is, when $\tau_{L} \gg \tau_{f}$. In this case, the fluctuations of supersaturation and droplet growth rate are highly correlated with the vertical velocity. Although the lowfrequency stochastic kinetic equations can explain some features of the drop size spectral dispersions in stratiform clouds, Manton (1979) showed that the stochastic approach leads to dispersions that are still narrower than those observed in convective clouds. By hypothesizing a negative correlation between the fluctuations in mean droplet radius and vertical velocity, Manton (1979) derived a modified version of the kinetic equation that produced broader (or bimodal) size spectra with dispersions increasing for a longer time. In a subsequent discussion of Manton's theory (Merkulovich and Stepanova 1981; Manton 1981), it was clarified that the condition of mass balance imposes some additional limitations on the basic assumptions and analytical solutions to the kinetic equation; in particular, it was shown that the bimodal spectra predicted by this theory should be monomodal in many cases, and the dispersions should be smaller. Austin et al. (1985) showed that the basic assumption of Manton's theory on the negative correlation between the fluctuations in integral radius and vertical velocity is not observed in the continental cumuli, while Curry (1986) found some evidence of negative correlation in Arctic stratus. Thus further verification or another hypothesis is required to break the link between supersaturation and vertical velocity, which appears to be the primary requirement for deriving a sufficiently broad drop spectra using the stochastic kinetic equations.

The first cloud models with explicit microphysics based on the kinetic equations were developed in the early 1970s (e.g., Clark 1973; Buikov and Pirnach 1973, Buikov and Khvorostyanov 1976). However, for many years explicit microphysical models were still a rather rare phenomenon among the much more frequently used bulk microphysical models. During 1980s and 1990s, the intensive development of cloud models with explicit microphysics was made possible by an increase in computational speed (e.g., Khvorostyanov 1982, 1995; Flossman et al. 1985; Marchuk et al. 1986; Tzivion et al. 1987; Kogan 1991; Feingold et al. 1994; Khvorostyanov and Sassen 1998). Additional impetus for explicit microphysics models has been provided by the Global Energy and Water Experiment (GEWEX) Cloud System Study, designed for development of the parameterizations for climate models (Browning 1994).

However, a gap remains between the theory of stochastic condensation and the practical needs of modeling. In applying the kinetic equation for stochastic condensation in numerical cloud models, we are faced with the following dilemmas.

1) A few attempts have been made to use the more complete stochastic kinetic equations in the low-frequency approximation of the kinetic equations in numerical cloud models (e.g., Vasilyeva et al. 1984), but the small characteristic timescale for the condensation process and presence of cross derivatives makes the numerical solution very computationally intensive. Hence, only the simplest versions of the kinetic equations in the high-frequency approximation are used now in most of the cloud models with explicit microphysics, neglecting terms that may influence the numerical solutions and physical effects. 2) Analytical solutions to stochastic kinetic equations 
in the low-frequency regime have been obtained of the Gaussian distribution type, which does not agree very well with observations that are typically in better agreement with a gamma distribution. This suggests that there are deficiencies in the formulation of the kinetic equations for stochastic condensation.

To address these concerns, we derive a new version of the kinetic equation for stochastic condensation for arbitrary relative values of $\tau_{L}$ and $\tau_{f}$ that is suitable for numerical cloud models. The principal assumptions that differentiate the new version of the kinetic equation from previous versions are 1) consideration of supersaturation as a nonconservative substance with differentiation between the macroscale and microscale supersaturation, and 2) consideration of the supersaturation fluctuations of various frequencies over the whole turbulent spectrum without assuming proportionality of supersaturation and vertical velocity. These assumptions break the link between the fluctuations in supersaturation and vertical velocity and lead to a solution of the gamma distribution type (see Part II of this paper, Khvorostyanov and Curry 1999a).

\section{A model of the condensation process in a turbulent cloud}

\section{a. Basic equations}

The kinetic equation of condensation can be written in the following form (following Buikov 1961; Levin and Sedunov 1966; Cotton and Anthes 1989; Khvorostyanov 1995):

$$
\frac{\partial f}{\partial t}+\frac{\partial}{\partial x_{i}}\left[\left(u_{i}-v(r) \delta_{i 3}\right) f\right]+\frac{\partial}{\partial r}(\dot{r} f)=J
$$

where $r$ is the droplet radius and $\dot{r}$ is the droplet radius rate of growth, $x_{i}$ and $u_{i}$ are the coordinates and velocity components, $J$ describes droplet sources and sinks, and the usual summation convention over doubled indices is assumed, $i=1,2,3$. Other variables are defined in the appendix. Equation (2.1) is a continuity equation for the dropsize spectra. The $\partial(\dot{r} f) / \partial r$ term in (2.1) represents the divergence of $f(r)$ due to condensation growth. Equation (2.1) can be regarded as considering a system in four-dimensional phase space $\left(x_{i}, r\right)$, where each possible state of the system corresponds to a uniquely determined point in the space $\left(x_{i}, r\right)$.

The rate of droplet growth, $\dot{r}$, is determined (following Sedunov 1974; Cotton and Anthes 1989) from

$$
\dot{r}=\frac{D S}{\Gamma \rho_{w} r} \quad \Gamma=1+\frac{L^{2}}{c_{p} R_{v} T^{2}} \frac{\rho_{s}}{\rho_{a}}=1+\frac{L}{c_{p}} \frac{\partial q_{s}}{\partial T},
$$

where $D$ is the vapor diffusion coefficient; $S=\rho_{v}-$ $\rho_{s}$ is the absolute supersaturation; $\rho_{w}, \rho_{a}, \rho_{v}$, and $\rho_{s}$ are the densities of water, air, vapor, and saturated vapor, respectively; $c_{p}$ is the specific heat capacity; $\Gamma$ is the psychrometric correction to the growth rate associated with the latent heat of condensation; $L$ is the latent heat of condensation; $R_{v}$ is the gas constant of water vapor; and $q_{s}$ is the saturated specific humidity. Equation (2.2) neglects for simplicity the solute (Raoult), kinetic (accomodation coefficient), and curvature (Kelvin) effects, but these effects can be easily incorporated.

The equation for supersaturation $S$ in the parcel can be derived from the heat balance equation and the continuity equation for water vapor (see, e.g., Khvorostyanov 1995; Khvorostyanov and Sassen 1998):

$$
\begin{aligned}
\frac{d T}{d t} & =-\gamma_{d} w+\frac{L}{c_{p} \rho_{a}} \varepsilon_{c}+Q_{\mathrm{rad}} \\
\frac{\partial \rho_{v}}{\partial t} & =-\frac{\partial\left(\rho_{v} u_{i}\right)}{\partial x_{i}}-\varepsilon_{c},
\end{aligned}
$$

where $\gamma_{d}=g / c_{p}$ is the dry adiabatic lapse rate, $w$ the vertical velocity, $Q_{\text {rad }}$ the radiative temperature change, and $\varepsilon_{c}$ the condensation rate, which can be written using (2.2) as

$$
\begin{aligned}
\varepsilon_{c} & =\int_{0}^{\infty} \frac{d m(r)}{d t} f(r)=4 \pi \rho_{w} \int_{0}^{\infty} r^{2} \dot{r} f(r) d r \\
& =\frac{S}{\Gamma \tau_{f}},
\end{aligned}
$$

where $m$ is the mass of the drop. Here $\tau_{f}$ is the characteristic condensation time that coincides with the supersaturation absorption time (called also phase relaxation time) analyzed by Squires (1952), Twomey (1959), Buikov (1963), Sedunov (1965, 1974), Mazin (1968), Austin et al. (1985), and many others and related to the droplet concentration $N$ and mean radius $\bar{r}$

$$
\begin{aligned}
\tau_{f} & =(4 \pi D N \bar{r})^{-1}, \quad N=\int_{0}^{\infty} f(r) d r \\
\bar{r} & =\int_{0}^{\infty} r f(r) d r .
\end{aligned}
$$

Here we present a modified version of the supersaturation equation in terms of the characteristic times that is more convenient for the further analysis. Starting from the equation $d S / d t=d \rho_{v} / d t-\left(d \rho_{s} / d T\right)(d T / d t)$, incorporating (2.3)-(2.5) and using also the mass continuity equation, the hydrostatic equation, and the ClausiusClapeyron equation, we obtain the supersaturation equation:

$$
\begin{aligned}
\frac{d S}{d t} & =-\Gamma \varepsilon_{c}+A w_{\text {ef }}=-\frac{S}{\tau_{f}}+A w_{\text {ef }} \\
A & =\frac{c_{p}}{L} \Gamma \rho_{a}\left(\gamma_{d}-\gamma_{s}\right),
\end{aligned}
$$

where $\gamma_{s}$ is the saturated adiabatic lapse rate and $w_{\text {ef }}$ is an effective vertical velocity (defined below). In the absence of the second term on the right-hand side of (2.6), 
the solution to (2.6) would be $S(t)=S(0) \exp \left(-t / \tau_{f}\right)$, thus the time $\tau_{f}$ determines the $e$-folding decrease of the initial supersaturation (note that it coincides with the characteristic condensation time defined above) and the first term on the right-hand side of (2.6) characterizes the rate of supersaturation absorption.

We have introduced in (2.6) an effective vertical velocity $w_{\text {ef }}=w+w_{\text {rad }}$, where $w_{\text {rad }}$ is the radiative-effective vertical velocity related to the radiative temperature change $Q_{\text {rad }}\left(\mathrm{K} \mathrm{s}^{-1}\right)$,

$$
w_{\mathrm{rad}}=-k_{\mathrm{rad}} Q_{\mathrm{rad}}, \quad k_{\mathrm{rad}}=\frac{\Gamma-1}{\Gamma\left(\gamma_{d}-\gamma_{s}\right)} \approx \frac{1}{\gamma d} .
$$

The advantage of introducing $w_{\text {rad }}$ is that it allows direct comparison of the dynamical and radiative sources of supersaturation generation. The maximum values $Q_{\mathrm{rad}}$ $\sim-10^{-3} \mathrm{~K} \mathrm{~s}^{-1}\left(-3.6 \mathrm{~K} \mathrm{~h}^{-1}\right.$ or $\left.-86 \mathrm{~K} \mathrm{day}^{-1}\right)$ and -2 $\times 10^{-3} \mathrm{~K} \mathrm{~s}^{-1}\left(-7.2 \mathrm{~K} \mathrm{~h}^{-1}\right)$ typical of Arctic stratus (Curry 1986), correspond to $w_{\text {rad }} \sim 10-20 \mathrm{~cm} \mathrm{~s}^{-1}$ in the upper cloud layer. Thus the magnitude of the radiativeeffective velocity can be comparable to the turbulent updrafts and is substantially larger than the synopticscale regular (mean) updraft velocity, providing the main source of condensation, instability, and turbulent kinetic energy production.

Equation (2.6) shows clearly that the rate of change of supersaturation is determined by the sources of supersaturation (updraft and radiative cooling) and the sink of supersaturation associated with condensation and inversely proportional to the phase relaxation time $\tau_{f}$. If quasi-stationary conditions are assumed in (2.6) $(d S / d t=0$, corresponding to the low-frequency approximation), the solution for equilibrium supersaturation, $S_{q}$, and for the relative $\tilde{S}_{q}=S_{q} / \rho_{s}$ is

$$
\begin{aligned}
& S_{q}=A w_{\mathrm{ef}} \tau_{f}=\frac{c_{p}\left(\gamma_{d}-\gamma_{s}\right)}{L 4 \pi D N \bar{r}} \Gamma \rho_{a} w_{\mathrm{ef}} \\
& \tilde{S}_{q}=\frac{c_{p}}{L} \frac{\left(\gamma_{d}-\gamma_{s}\right)}{4 \pi D N \bar{r}} \frac{\Gamma}{q_{s}} w_{\mathrm{ef}} .
\end{aligned}
$$

\section{b. Stochastic equations}

The kinetic equation (2.1), the droplet growth equation (2.2), and the supersaturation equation (2.6) close the system of equations required to determine the evolution of the dropsize distribution if the velocity, temperature, and humidity fields are specified. To account for turbulence and other fluctuations, we represent all quantities as sums of mean (denoted by overbar) and fluctuating (denoted by prime) values:

$$
\begin{array}{ll}
u=\bar{u}+u^{\prime}, & f=\bar{f}+f^{\prime}, \\
S=\bar{S}+S^{\prime}, & \dot{r}=\bar{r}+r^{\prime},
\end{array}
$$

where the mean refers to an ensemble average. Substituting (2.10) into (2.1) and averaging using the Reynolds procedure, we derive an equation for the drop size spectra that is averaged over the ensemble of realizations

$$
\begin{aligned}
\frac{\partial \bar{f}}{\partial t} & +\frac{\partial}{\partial x_{i}}\left(\overline{u_{i}} \bar{f}\right)+\frac{\partial}{\partial r}(\overline{\dot{r}} \bar{f}) \\
& =-\frac{\partial}{\partial x_{i}} \overline{u_{i}^{\prime} f^{\prime}}-\frac{\partial}{\partial r} \overline{\dot{r}^{\prime} f^{\prime}}+\bar{J} .
\end{aligned}
$$

Henceforth we shall omit the bars over the mean values, other than for the covariance terms. The nucleation term $\bar{J}$ can be calculated by appropriate averaging of the CCN activity spectra (e.g., Twomey 1959; Sedunov 1974; Khvorostyanov and Curry 1999b); the detailed consideration of $\bar{J}$ is beyond the scope of this paper. Note that the averaging here can be understood also in the context of averaging over the grid scale of a large eddy simulation (LES) model, time, or grid-volume averaging, with the corresponding requirements on the averaging procedure (see Cotton and Anthes 1989, chapter $3)$.

In the case of the grid-volume average with the scale $L_{x,}$ the average value of any variable is

$$
\bar{\psi}=\left(1 / L_{x}\right) \int_{0}^{L_{x}} \psi d x .
$$

For typical LES models or 1-Hz aircraft sampling distance, $L_{x} \sim 50-100 \mathrm{~m}$. Averaging over this spatial scale implies filtering only of the highest frequencies of motion up to $\omega_{\max } \sim 1 \mathrm{~Hz}$, while the peak of the turbulent energy may lie near the values of $\omega_{\max }$ (e.g., Curry et al. 1988). Hence, the average quantities defined above still contain turbulent fluctuations at longer wavelengths with $\omega<\omega_{\max }$, and correlations still exist between the average values and fluctuations denoted by primes (see Cotton and Anthes 1989, section 3.6). This should be kept in mind when defining the "mean" supersaturation $\bar{S}$, mean vertical velocity $\bar{w}_{\text {ef }}$, and their relation. In this case, mean values implies grid averages.

The right-hand side of (2.11) is ascribed a stochastic meaning, since $u$ and $\dot{r}$ (which depends on supersaturation fluctuations) vary randomly. We represent the fluctuations $f^{\prime}$ in a form analogous to the Prandtl mixing length concept with a generalization to the $4 \mathrm{D}$ phase space $\left(x_{j}, r\right)$ :

$$
f^{\prime}=-l_{j}^{\prime} \frac{\partial f}{\partial x_{j}}-\frac{\partial}{\partial r} l_{r}^{\prime} f .
$$

The first term on the right-hand side of (2.12) corresponds to the mixing of a conservative passive scalar, where $l_{j}^{\prime}$ is the Prandtl mixing pathlength. The second term arises from the nonconservativeness of $f$, and the Prandtl mixing length concept is extended to the $r$ dimension in the phase space following Voloshchuk and Sedunov (1977).

If $\tau_{f} \ll \tau_{L}$ (the low-frequency regime), then the fluctuations of $\dot{r}^{\prime}$ can be determined from the Maxwellian 
growth rate equation. Fluctuations in droplet growth rate can be determined as

$$
\dot{r}^{\prime}=\frac{D S^{\prime}}{\Gamma \rho_{w} r} .
$$

If the fluctuations $S^{\prime}$ are determined from (2.9), we have

$$
S^{\prime}=A w_{\mathrm{ef}}^{\prime} \tau_{f}=\frac{c_{p}}{L} \Gamma \rho_{a} \frac{\left(\gamma_{d}-\gamma_{s}\right)}{4 \pi D N \bar{r}} w_{\mathrm{ef}}^{\prime}
$$

Substituting (2.9), (2.12), (2.13), (2.14), into (2.11) would yield the previous forms of stochastic kinetic equation (as discussed in the introduction) that are valid in the low-frequency regime, with the additional assumption that both mean supersaturation and its fluctuations are proportional to the corresponding vertical velocity. The assumption of low frequency is implicit in (2.14) because fluctuations of supersaturation are correlated exactly with the fluctuations of vertical velocity.

\section{c. Supersaturation fluctuations}

As noted in the introduction, the basic assumption used previously in derivations of the stochastic kinetic equation, that $\bar{S}$ and $S^{\prime}$ are both related to the respective components of the vertical velocity, leads to the size distribution of Gaussian type that is too narrow in comparison to the observations. Thus we hypothesize and seek alternative relationships to (2.13), (2.14) that are not based on the assumption of proportionality of fluctuations of growth rate and vertical velocity and account for the different growth rates in fluctuations than in regular (low frequency) growth.

Supersaturation fluctuations can be associated with turbulent fluctuations in temperature and humidity, which may or may not be associated with vertical velocity. There is a hierarchy of spatial scales of turbulent motions in the cloud, with the larger eddy sizes having a greater coherence with supersaturation. Additional supersaturation fluctuations may occur on the drop microscale that are unrelated to vertical velocity fluctuations but might be induced by other turbulent fluctuations such as those caused by the mixing of the cloudy parcels with different properties that act to break the link between vertical velocity and supersaturation.

Suppose a supersaturation fluctuation $S^{\prime}$ arises in a cloudy parcel due to a turbulent fluctuation. Vapor begins to flow to the droplets and unless the equilibrium is attained in the volume $V_{L}$ in the vicinity of each droplet, there will be a nonstationary vapor concentration field $\rho_{v i}(r)$. To calculate these vapor fields, we need to solve a complex system of many diffusion equations for $\rho_{v i}(r)$ from all drops that influence each other in order to calculate a self-consistent vapor field formed by the superposition of the overlapping fields from individual drops (Sedunov 1974; Srivastava 1989). The exact solution to this complicated diffusion problem has not been found; thus various approximate methods are used.

Many models of condensation growth were considered over the last two decades that may help to break the link between $S^{\prime}$ and $w^{\prime}$. The common feature of several of them is that they predict the local growth of supersaturation fluctuations in proportion to the droplet radius, $S^{\prime} \sim r$. Srivastava (1989) introduced the concept of a microscopic supersaturation fluctuations, which can vary from drop to drop due to randomness in the droplet spatial distribution; the main part of this microscopic supersaturation $S^{\prime}$ is proportional to the droplet radius.

The inhomogeneous mixing theory of Baker and Latham (1979) and Baker et al. (1984), based on the chamber mixing experiments by Latham and Reed (1977), predicts the local increase of supersaturation in the vicinity of the larger drops that are less affected by mixing with entrained dry air, because the newly activated smaller droplets (that form later in the areas of complete evaporation) cannot compete as effectively for the available water vapor. Thus the effective supersaturation in the inhomogeneous mixing theory also increases with the drop radius.

Further evidence for the proportionality $S^{\prime} \sim r$ for small timescales comes from the detailed calculations of droplet growth that account for the kinetic correction (Fukuta and Walter 1970) and the concept of the modified diffusion coefficient, $D_{m}(r)$, which accounts for this correction (Pruppacher and Klett 1997, p. 506). Calculations of droplet growth show that the magnitude of the kinetic correction depends on time and drop mass. Over short time periods comparable to $\tau_{f}(10-20 \mathrm{~s})$, the kinetic correction causes a strong suppression of the growth of the smaller droplets. The value of $D_{m}(r) \sim$ $r$ increases almost linearly with radius for small droplets (Pruppacher and Klett 1997, Table 13.1). Over periods much longer than $\sim 20 \mathrm{~s}$, the kinetic correction becomes negligible and Maxwellian growth occurs. We performed similar calculations of supersaturation during relaxation $(\sim 3-10 \mathrm{~s})$ with account for the curvature and kinetic corrections with various accomodation coefficients (0.04-1), which also showed that the effective supersaturation, $S^{\prime}(r, t)$, increases with radius such that it can be roughly approximated by a linear dependence, $S^{\prime}(r, t) \sim \alpha(t) r$. The slope $\alpha(t)$ decreases with time; thus the dependence of $S^{\prime}$ on $r$ weakens and the usual Maxwellian growth takes place for the larger times. Thus accounting for the kinetic and curvature corrections leads to the quasi-kinetic regime of growth for short timescales comparable to the phase relaxation time $\tau_{f}$ and the diffusion regime during long timescales.

Since we do not account explicitly here for the above curvature and kinetic corrections, activation, and other effects that lead to the dependence $S^{\prime} \sim r$ (i.e., suppression of the growth of smaller droplets), we consider an approximate model of condensational growth in supersaturation fluctuations. We characterize the average rate of supersaturation absorption, $\dot{S}_{i}^{\prime}$, by the $i$ th drop 
from its vicinity, $V_{L} \sim N^{-1}$, by using the local relaxation time $\tau_{f i}=\left(4 \pi D N r_{i}\right)^{-1}$ as a scaling time, similar to the total relaxation time for the ensemble of drops (2.5). Then $\dot{S}_{i}^{\prime}$ can be written approximately as

$$
\dot{S}_{i}^{\prime} \approx S^{\prime} / \tau_{f i}=S^{\prime}\left(4 \pi D N r_{i}\right)
$$

so that the rate of absorption of supersaturation fluctuation by a drop increases with increasing radius. Now, the supersaturation $S_{i}^{\prime}$ absorbed by the $i$ th drop during the entire period of relaxation in the ensemble of the drops is proportional to the total relaxation time, $\tau_{f}$. Using (2.16) and (2.5) we can determine

$$
\dot{S}_{i}^{\prime}=\dot{S}_{i}^{\prime} \tau_{f}=S^{\prime} \frac{\tau_{f}}{\tau_{f i}}=S^{\prime} \frac{4 \pi D N r_{i}}{4 \pi D N \bar{r}}=S^{\prime} \frac{r_{i}}{\bar{r}} .
$$

So, the effective microscale supersaturation in (2.17) is approximated during relaxation as a linear function of drop radius. Incorporation of (2.17) into (2.13) for $\dot{r}_{i}^{\prime}$ yields the desired result

$$
\dot{r}^{\prime}=\frac{D S^{\prime}}{\Gamma \rho_{w} \bar{r}}=\phi S^{\prime} \quad \phi=\frac{D}{\Gamma \rho_{w} \bar{r}} .
$$

The physical meaning of (2.16)-(2.18) is that due to suppressed growth of the smaller drops, the effective relaxation rate of supersaturation is faster for the larger droplets, so during relaxation the larger droplets absorb the larger fraction of the initial supersaturation fluctuation $S^{\prime}$. Equation (2.18) shows that fluctuation of the growth rate described by this model is independent of radius, which is equivalent to kinetic regime in fluctuations. Another important feature of (2.18) is that we do not assume here $S^{\prime} \sim w^{\prime}$ as in previous theories, but simply consider $S^{\prime}$ as a stochastic variable.

Support for our model of microscale condensation is provided by several previous studies, as discussed above. After substituting into (2.13) the effective diffusivity $D_{m}(r) \sim r$, which can approximate the results by Fukuta and Walter (1970) and Pruppacher and Klett (1997, Table 13.1), we also arrive at a formulation (2.18) of $\dot{r}^{\prime}$ that is independent of radius.

From (2.18), we can infer that the droplet spectrum during $\Delta t$ in the kinetic regime would be displaced as a whole by $\Delta r=\varphi S_{0}^{\prime} \Delta t$, whereby the displacement is independent of radius and the shape of the spectrum is preserved. The possibility of such a spectral shift was noted by Baker et al. (1984) when analyzing the consequences of the Broadwell-Breidenthal (1982) model.

Hence, we have developed a model of condensation in a turbulent medium that is naturally separated by scales: 1) the usual Maxwellian diffusion regime controls condensational growth for longer timescales as for the regular condensation by using (2.1) for $\dot{r}$ [with use of calculated values of supersaturation or equilibrium supersaturation $S_{q}$ (2.9) for the steady state]; and 2) droplet growth occurs in the kinetic regime (2.17), (2.18) for small timescales in fluctuations. Therefore fluctuations in supersaturation, $S^{\prime}$, and growth rate, $\dot{r}^{\prime}$, have a stochastic meaning, but they are not proportional to the vertical velocity fluctuation $w^{\prime}$ as in the lowfrequency approximation. This model therefore breaks the link between the fluctuations in $S^{\prime}$ (or growth rate) and $w^{\prime}$.

By integrating (2.18) over the duration of a single fluctuation $\left(\sim \tau_{f}\right)$, an expression for $l_{r}^{\prime}$ is derived that determines an analog of Prandtl's mixing length in radii space:

$$
l_{r}=\int_{0}^{t} \dot{r}^{\prime} d t^{\prime}=\phi \int_{0}^{t} S^{\prime}\left(t^{\prime}\right) d t^{\prime} .
$$

As (2.19) is independent of radius, (2.12) for $f^{\prime}$ then becomes

$$
f^{\prime}=-l_{j}^{\prime} \frac{\partial f}{\partial x_{j}}-l_{r}^{\prime} \frac{\partial f}{\partial r} .
$$

Equations (2.17)-(2.20) allow evaluation of the covariances in the kinetic equation (2.11), which is done in the next section.

\section{Evaluation of correlation functions}

In this section, we use concepts from the statistical theory of turbulence (e.g., Monin and Yaglom 1967) to evaluate the correlation functions $\overline{u_{i}^{\prime} f^{\prime}}$ and $\overline{\dot{r}^{\prime} f^{\prime}}$ in (2.11). We assume that the velocity fluctuation vectors $u_{j}^{\prime}$, the supersaturation $S_{j}^{\prime}$, and mixing lengths $l_{j}^{\prime}$ are random quantities, which we represent as expansions in form of Fourier-Stieltjes integrals:

$$
\begin{aligned}
& u_{j}^{\prime}(t)=\int_{-\infty}^{\infty} e^{i \omega t} d u^{\prime}(\omega) \quad S^{\prime}(t)=\int_{-\infty}^{\infty} e^{i \omega t} d S^{\prime}(\omega) \\
& l_{j}^{\prime}(t)=\int_{0}^{t} u_{j}^{\prime}\left(t_{1}\right) d t_{1}=\int_{0}^{t} d t_{1} \int_{-\infty}^{\infty} e^{i \omega t_{1}} d u_{j}^{\prime}(\omega),
\end{aligned}
$$

where $\omega$ is the frequency of turbulent spectrum, and the symbol $t_{1}$ is used hereafter to denote the time variable of integration but not the fluctuation and superscript $i$ is $\sqrt{-1}$.

The complex amplitudes $d u_{j}^{\prime}(\omega), d S^{\prime}(\omega)$ have an analytic continuation into the region $\omega<0$, with $d u_{j}^{\prime}(-\omega)$ $=d u_{j}^{\prime} *(\omega)$ and are normalized to the Dirac delta function, $\delta(\omega)$, and a spectral function $F_{i j}(\omega)$ (following Monin and Yaglom 1967):

$$
\begin{aligned}
\overline{d u_{i}^{\prime}(\omega) d u_{j}^{\prime *}\left(\omega^{\prime}\right)} & =F_{i j}(\omega) \delta\left(\omega-\omega^{\prime}\right) d \omega d \omega^{\prime} \\
F_{i j}(\omega) & =\frac{1}{2 \pi} \int_{0}^{\infty} e^{-i \omega t} B_{i j}(t) d t .
\end{aligned}
$$

Assuming stationary, locally homogeneous, isotropic turbulence, we can also define (following Monin and Yaglom 1967) the velocity correlation function $B_{i j}$ and the turbulence diffusion coefficient tensor $k_{i j}$, 


$$
\begin{aligned}
B_{i j}\left(t-t_{1}\right) & =\int_{-\infty}^{\infty} e^{i \omega\left(t-t_{1}\right)} F_{i j}(\omega) d \omega \\
& =\int_{0}^{\infty} \cos \left[\omega\left(t-t_{1}\right)\right]\left[2 F_{i j}(\omega)\right] d \omega \\
k_{i j} & =\int_{0}^{\infty} B_{i j}(\tau) d \tau,
\end{aligned}
$$

where $\tau=t-t_{1}$, and $F_{i j}(\omega)$ is related to the frequently used spectral density $E_{i f}(\omega)$ defined at positive $\omega>0$ as $2 F_{i j}(\omega)=E_{i j}(\omega)$.

The covariance $\overline{l_{i}^{\prime} u_{j}^{\prime}}$ is readily determined from (3.1) to be

$$
\begin{aligned}
\overline{l_{i}^{\prime} u_{j}^{\prime}} & =\int_{0}^{t} d t_{1} \int_{-\infty}^{\infty} e^{i \omega t} \int_{-\infty}^{\infty} e^{-i \omega^{\prime} t_{1}} \overline{d u_{i}^{\prime}(\omega) d u_{j}^{*}\left(\omega^{\prime}\right)} \\
& =\int_{0}^{t} d t_{1} B_{i j}\left(t-t_{1}\right) .
\end{aligned}
$$

\section{a. Supersaturation as a nonconservative variable}

Determination of covariances involving supersaturation is complicated by the fact that supersaturation is nonconservative because of turbulent fluctuations of temperature and humidity, and of phase changes when $S \neq 0$. Nonconservativeness of supersaturation requires introduction of nonconservative covariances and turbulence coefficients.

Separating fluctuations from the average quantities in the supersaturation equation (2.6), we can write

$$
\frac{d S^{\prime}}{d t}=-\frac{S^{\prime}}{\tau_{f}}+A u_{3}^{\prime} .
$$

By substituting (3.1) into (3.5), we can determine a spectral analog of the supersaturation equation:

$$
\begin{aligned}
\int_{-\infty}^{\infty} e^{i \omega t}(i \omega) d S^{\prime}(\omega)= & -\frac{1}{\tau_{f}} \int_{-\infty}^{\infty} e^{i \omega t} d S^{\prime}(\omega) \\
& +A \int_{-\infty}^{\infty} e^{i \omega t} d u_{3}^{\prime}(\omega) \\
d S^{\prime}(\omega)= & \frac{A}{i\left(\omega-i \omega_{p}\right)} d u_{3}^{\prime}(\omega)
\end{aligned}
$$

where $\omega_{p}=\tau_{f}^{-1}=4 \pi D N \bar{r}$ is the imaginary part of the frequency, which can be called the "supersaturation relaxation frequency." The complex frequency in the denominator of (3.6b) with imaginary part $\omega_{p}>0$ reflects the nonconservativeness of the supersaturation and is written in the form similar to that in electrodynamics for the wave propagation in an absorbing media.

If we assume in (3.6b) that $\omega \ll \omega_{p}$ (low-frequency approximation), the amplitude $d S^{\prime}(\omega)$ becomes proportional to the vertical velocity $d u^{\prime}(\omega)$ at all frequencies, and we come to the previous formulations of stochastic theory by Levin and Sedunov (1966), Voloshchuk and Sedunov (1977), and Manton (1979). Thus our approach generalizes the previous theories by use of (3.6b), which breaks the links between supersaturation and vertical velocity. In deriving (3.6) we assumed for simplicity that $\tau_{f}=\omega_{p}^{-1}$ are constant; all subsequent manipulations can be readily generalized to allow for fluctuations of $\tau_{f}$ [e.g., Voloshchuk and Sedunov (1977) for the lowfrequency regime; Cooper (1989)]; however, as was noted in section $2 \mathrm{c}$, assumption of $\tau_{f} \approx$ const is a good approximation when considering supersaturation relaxation on the microscale over timescales $t \sim \tau_{f}$.

\section{b. Covariances with supersaturation}

By incorporating (3.6), (3.1), and (3.2), we obtain the following expression for the correlation function $\overline{u_{j}^{\prime} S^{\prime}}$ :

$$
\begin{aligned}
& \overline{S^{\prime}(t) u_{j}^{\prime}\left(t_{1}\right)} \\
& =\int_{-\infty}^{\infty} e^{i \omega t} \int_{-\infty}^{\infty} e^{-i \omega^{\prime} t_{1}} \frac{A}{i\left(\omega-i \omega_{p}\right)} \overline{d u_{3}^{\prime}(\omega) d u_{j}^{\prime *}\left(\omega^{\prime}\right)} \\
& =\delta_{i 3}\left(A / \omega_{p}\right) B_{i j}^{n}\left(t-t_{1}, \tau_{f}\right),
\end{aligned}
$$

where we introduced the correlation function of velocity with supersaturation as a nonconservative substance (index " $n$ ") with a characteristic "nonconservativeness" time $\tau_{f}=\omega_{p}^{-1}$ :

$$
\begin{aligned}
B_{i j}^{n}\left(t, \tau_{f}\right) & =\int_{-\infty}^{\infty} e^{i \omega t} \frac{\omega_{p}}{i\left(\omega-i \omega_{p}\right)} F_{i j}(\omega) d \omega \\
& =\int_{0}^{\infty} \frac{\left[\omega_{p}+\omega \tan \omega t\right]}{1+\left(\omega / \omega_{p}\right)^{2}} \cos \omega t\left[2 F_{i j}(\omega)\right] d \omega .
\end{aligned}
$$

The first term in subintegral function of (3.8b) determines the nonconservative effects and the rest coincides with the expansion of the conservative function (3.3). We can derive analogously the turbulence diffusion coefficient tensor for a nonconservative substance,

$$
k_{i j}^{n}\left(\tau_{f}\right)=\int_{0}^{\infty} B_{i j}^{n}\left(\tau, \tau_{f}\right) d \tau .
$$

Following an approach analogous to the derivation of (3.7), we can obtain an expression for the supersaturation autocorrelation function

$$
\overline{S^{\prime}(t) S^{\prime}\left(t_{1}\right)}=\int_{-\infty}^{\infty} e^{i \omega t} \int_{-\infty}^{\infty} e^{i \omega^{\prime} t_{1}} \frac{A^{2}}{\left(i \omega+\omega_{p}\right)\left(-i \omega+\omega_{p}\right)} \overline{d u_{3}^{\prime}(\omega) d u_{3}^{\prime *}\left(\omega^{\prime}\right)}=\left(A / \omega_{p}\right)^{2} \delta_{i 3} \delta_{j 3} B_{i j}^{n n}\left(t-t_{1}, \tau_{f}\right),
$$


where we introduce the autocorrelation function of a nonconservative substance and the corresponding turbulence coefficient:

$$
\begin{aligned}
B_{i j}^{n n}\left(t, \tau_{f}\right) & =\int_{-\infty}^{\infty} e^{i \omega t} \frac{\omega_{p}^{2}}{\left(\omega^{2}+\omega_{p}^{2}\right)} F_{i j}(\omega) d \omega \\
& =\int_{0}^{\infty} \frac{2 \cos \omega t}{1+\left(\omega / \omega_{p}\right)^{2}} F_{i j}(\omega) d \omega \\
k_{i j}^{n n}\left(\tau_{f}\right) & =\int_{0}^{\infty} B_{i j}^{n n}\left(t, \tau_{f}\right) d t .
\end{aligned}
$$

\section{c. Covariances with drop size distribution function}

For the general case of arbitrary relations between $\tau_{f}$ and $\tau_{L}$, making use of (2.19), (2.20), (3.4), and (3.8), we obtain for the correlation function $\overline{u_{i}^{\prime} f^{\prime}}$ in (2.11)

$$
\begin{aligned}
\overline{u_{i}^{\prime} f^{\prime}}=-\int_{0}^{t} d t_{1}[ & B_{i j}\left(t-t_{1}\right) \frac{\partial f\left(t_{1}\right)}{\partial x_{j}} \\
& \left.+B_{i j}^{n}\left(t-t_{1}, \tau_{f}\right) \delta_{j 3} G \frac{\partial f\left(t_{1}\right)}{\partial r}\right],
\end{aligned}
$$

where we have introduced the parameter $G$,

$$
G=\varphi A \tau_{f}=\frac{D}{\rho_{w} \bar{r}} \frac{c_{p}}{L} \frac{\gamma_{d}-\gamma_{s}}{4 \pi D N \bar{r}} \rho_{a}=\frac{\rho_{a}}{\rho_{w}} \frac{c_{p}}{L} \frac{\gamma_{d}-\gamma_{s}}{4 \pi N \bar{r}^{2}} .
$$

From (2.19), (2.20), (3.7), and (3.10), we obtain for $\overline{\dot{r}^{\prime} f^{\prime}}$

$$
\begin{gathered}
\overline{\dot{r}^{\prime} f^{\prime}}=\varphi \overline{S^{\prime} f^{\prime}} \\
=-\varphi \int_{0}^{t} d t_{1}\left[\overline{S^{\prime}(t) u_{j}^{\prime}\left(t_{1}\right)} \frac{\partial f\left(t_{1}\right)}{\partial x_{j}}+\varphi \overline{S^{\prime}(t) S^{\prime}\left(t_{1}\right)} \frac{\partial f\left(t_{1}\right)}{\partial r}\right] \\
=-\int_{0}^{t} d t_{1}\left[G \delta_{i 3} B_{i j}^{n}\left(t-t_{1}, \tau_{f}\right) \frac{\partial f\left(t_{1}\right)}{\partial r}\right. \\
\left.+G^{2} \delta_{i 3} \delta_{j 3} B_{i j}^{n n}\left(t-t_{1}, \tau_{f}\right) \frac{\partial f\left(t_{1}\right)}{\partial r}\right] .
\end{gathered}
$$

Equations (3.12) and (3.14) are integrated following Stepanov (1975). The main contribution to the integrals comes from the region $t-t_{1} \sim \tau_{L}$, because the correlation functions decrease rapidly beyond this interval. During the time period $t-t_{1} \sim \tau_{L}$, the distribution function $f$ varies much slower than the correlation functions and we can remove $\partial f / \partial x$ and $\partial f / \partial r$ from the integrals and simplify (3.12) and (3.14) to

$$
\begin{aligned}
\overline{u_{i}^{\prime} f^{\prime}}= & -\frac{\partial f}{\partial x_{j}} \int_{0}^{t} d t_{1} B_{i j}\left(t-t_{1}\right) \\
& -\delta_{j 3} G \frac{\partial f}{\partial r} \int_{0}^{t} d t_{1} B_{i j}^{n}\left(t-t_{1}, \tau_{f}\right) \text { and }
\end{aligned}
$$

$$
\begin{aligned}
\overline{\dot{r}^{\prime} f^{\prime}}= & -\delta_{i 3} G \frac{\partial f}{\partial x_{j}} \int_{0}^{t} d t_{1} B_{i j}^{n}\left(t-t_{1}, \tau_{f}\right) \\
& -\delta_{i 3} \delta_{j 3} G^{2} \frac{\partial f}{\partial r} \int_{0}^{t} d t_{1} B_{i j}^{n n}\left(t-t_{1}, \tau_{f}\right) .
\end{aligned}
$$

The integrals in (3.15) and (3.16) can then be evaluated from $t=0$ to $\infty$ since the integrated functions decrease rapidly with time beyond $\tau_{L}$. We can write (3.15) and (3.16) by including the definitions of the turbulence coefficients (3.3), (3.9), and (3.11) as

$$
\begin{aligned}
& \overline{u_{i}^{\prime} f^{\prime}}=-k_{i j} \frac{\partial f}{\partial x_{j}}-\delta_{j 3} k_{i j}^{n}\left(\tau_{f}\right) G \frac{\partial f}{\partial r} \\
& \overline{\dot{r}^{\prime} f^{\prime}}=-\delta_{i 3} k_{i j}^{n}\left(\tau_{f}\right) G \frac{\partial f}{\partial x_{j}}-\delta_{i 3} \delta_{j 3} k_{i j}^{n n}\left(\tau_{f}\right) G^{2} \frac{\partial f}{\partial r} .
\end{aligned}
$$

For high-frequency turbulence, only the first term of the expression for $\overline{u_{i}^{\prime} f^{\prime}}$ on the right-hand side of (3.17) and (3.18) remain since the nonconservative turbulence coefficients vanish. In the low-frequency approximation $\omega$ $\ll \omega_{p}\left(\tau_{L} \gg \tau_{f}\right)$, the term $\omega_{p} /\left[i\left(\omega-i \omega_{p}\right)\right]$ in (3.8a) tends to unity and the supersaturation-velocity correlation function reduces to (3.3), so $B_{i j}^{n}=B_{i j}$, and $k_{i j}^{n}=$ $k_{i j}$. It follows also from (3.11) that $B_{i j}^{n n}=B_{i j}, k_{i j}^{n n}=k_{i j}$; that is, nonconservative effects (dependence of the correlation functions and turbulence coefficients on the supersaturation relaxation time) vanish in the low-frequency regime. In the high-frequency approximation $\omega$ $\gg \omega_{p}\left(\tau_{f} \gg \tau_{L}\right)$, we find from (3.7)-(3.11) that $B_{i j}^{n}=$ $0, k_{i j}^{n}=0, B_{i j}^{n n}=0, k_{i j}^{n n}=0$. In liquid clouds, the value of $\tau_{f} \sim 1-10$ s (Sedunov 1974), while $\omega \sim 0.2-1 \times 10^{-2}$ $\mathrm{s}^{-1}$ and $\tau_{L} \sim 100-600 \mathrm{~s}$ (e.g., Curry et al. 1988), which is closer to the low-frequency approximation; hence the high-frequency kinetic equation used now in most cloud models with explicit microphysics is oversimplified. In crystalline clouds such as cirrus or "diamond dust," for which this technique can be also applied, the concentration of particles can be by 1-3 orders of magnitude less than in liquid stratus, and the values of $\tau_{f}$ can be 10-180 min (Khvorostyanov and Sassen 1998), while $\tau_{L} \sim 5-15 \mathrm{~min}$. Thus the values of $\tau_{f}$ and $\tau_{L}$ can be comparable or the situation can be closer to the highfrequency regime, which should influence $k_{n}, k_{n n}$. In particular, if the maximum in the turbulent spectrum is located near $\omega \sim \tau_{L}^{-1}$, and $\tau_{f} \sim \tau_{L}$, then (3.8b) and (3.11) show that $k_{n}, k_{n n} \sim 0.5 k$; these values will decrease with increasing $\tau_{f}$. Equations (3.8) and (3.11) allow calculations of the nonconservative correlation functions and turbulent coefficients either with various theoretical models of the turbulent spectra or directly with the observed spectra with appropriate choice of the limits in the integrals.

\section{General kinetic equations of stochastic condensation}

Substituting Eqs. (3.17) and (3.18) into (2.11) and assuming that the droplet growth rate $\dot{r}$ is determined 
from (2.1), we obtain the general kinetic equation of stochastic condensation:

$$
\begin{aligned}
\frac{\partial f}{\partial t}+ & \frac{\partial}{\partial x_{i}}\left[\left(u_{i}-v(r) \delta_{i 3}\right) f\right]+\frac{\partial}{\partial r}\left(\frac{b S}{r} f\right) \\
= & \frac{\partial}{\partial x_{i}} k_{i j} \frac{\partial f}{\partial x_{j}}+\frac{\partial}{\partial x_{i}} \delta_{j 3} k_{i j}^{n} G \frac{\partial f}{\partial r}+\delta_{i 3} k_{i j}^{n} G \frac{\partial^{2} f}{\partial x_{i} \partial r} \\
& +\delta_{i 3} \delta_{j 3} k_{i j}^{n n} G^{2} \frac{\partial^{2} f}{\partial r^{2}}+\bar{J}
\end{aligned}
$$

where $b=D /\left(\Gamma \rho_{a}\right)$. Recall that all the quantities on the left-hand side $\left(f, u_{i}, w, S\right)$ indicate averaged values (in the sense discussed in section 2b), and all fluctuations are included on the right-hand side of the equation.

If the scale of averaging (e.g., over a grid box of a numerical model) is chosen to be sufficiently large so that the characteristic time of averaging is much larger than $\tau_{f}$, then the mean supersaturation $\bar{S}$ can be equal to the equilibrium value $S_{q}$ related in (2.9) to the mean vertical velocity $\bar{w}_{\text {ef }}$. Note, however, that when using this representation for $\bar{S}$, the value of $\bar{w}_{\text {ef }}$ should not be understood as simply the mean (synoptic scale) vertical velocity.

This $\bar{w}_{\text {ef }}$ should represent an "effective" subgrid-averaged vertical velocity. Since the grid-box average still contain turbulent fluctuations of the larger scales (see section $2 \mathrm{~b}$ ), and correlations still exist between smallerscale fluctuations (primes) and mean values (overbars), the effective subgrid average $\bar{w}_{\text {ef }}$ can be estimated from the relation $\bar{w}_{\text {ef }} \cdot \bar{f} \sim \overline{w^{\prime} f^{\prime}}$. Multiplying this relation by (4/3) $\pi \rho_{w} r^{3}$ and integrating over radii, we obtain a relation $\bar{w}_{\text {ef }} \sim \overline{w^{\prime} q_{L}^{\prime}} / q_{L}$, which allows estimation of $\bar{w}_{\text {ef }}$ from the measurements of $q_{L}$ and the covariance of the fluctuations of the vertical velocities and $q_{L}, \overline{w^{\prime} q_{L}^{\prime}}$.

Substituting the equilibrium value $S_{q}$ (2.9) into (4.1), we obtain

$$
\begin{aligned}
\frac{\partial f}{\partial t}+ & \frac{\partial}{\partial x_{i}}\left[\left(u_{i}-v(r) \delta_{i 3}\right) f\right]+\frac{\partial}{\partial r}\left(\frac{c}{r} w_{\mathrm{ef}} f\right) \\
= & \frac{\partial}{\partial x_{i}} k_{i j} \frac{\partial f}{\partial x_{j}}+\frac{\partial}{\partial x_{i}} \delta_{j 3} k_{i j}^{n} G \frac{\partial f}{\partial r}+\delta_{i 3} k_{i j}^{n} G \frac{\partial^{2} f}{\partial x_{i} \partial r} \\
& +\delta_{i 3} \delta_{j 3} k_{i j}^{n n} G^{2} \frac{\partial^{2} f}{\partial r^{2}}+\bar{J} \\
c= & \frac{D A \tau_{f}}{\rho_{w} \Gamma}=D \frac{\rho_{a}}{\rho_{w}} \frac{c_{p}}{L} \frac{\left(\gamma_{d}-\gamma_{s}\right)}{4 \pi D N \bar{r}} .
\end{aligned}
$$

The representation (4.1a) may be suitable for models with grid boxes a few tens or hundred meters or larger and/or for processes where the mean supersaturation is close to the quasi-steady value, while the more complete form (4.1) is preferable for models with finer resolution (e.g., on the smaller scales of LES models), or for pro- cesses or cloud layers where supersaturation is not quasi-steady. Note that the minimum scales of averaging and conditions when $S \approx S_{q}$ can be determined probably only with the use of the LES models with fine resolution after detailed analysis of the entire supersaturation field.

To represent the kinetic equation in a more compact form, we adopt a methodology from quantum field theory that uses left and right operators. We introduce the left operator $\hat{H}_{L}$ and the right operator $\hat{H}_{R}$, such that

$$
\hat{H}_{L} k_{i j}=k_{i j}^{n}, \quad k_{i j}^{n}=k_{i j} \hat{H}_{R}, \quad \hat{H}_{L} k_{i j} \hat{H}_{R}=k_{i j}^{n n} .
$$

The left operator $\hat{H}_{L}$ acts to the right and the right operator $\hat{H}_{R}$ acts to the left. The operators convert the conservative tensor, $k_{i j}$, into a nonconservative tensor, $k_{i j}^{n}$, and commute with operators $\partial / \partial x_{i}$ and $\partial / \partial r$. Further, $\hat{H}_{L, R}$ are integral operators that convert the correlation function (3.3) into (3.8), (3.11).

Incorporating these operators into the right-hand side of (4.1) and (4.1a), we obtain

$$
\begin{aligned}
& \frac{\partial f}{\partial t}+\frac{\partial}{\partial x_{i}}\left[\left(u_{i}-v(r) \delta_{i 3}\right) f\right]+\frac{\partial}{\partial r}\left(\frac{b S}{r} f\right) \\
& =\left(\frac{\partial}{\partial x_{i}}+\delta_{i 3} G \hat{H}_{L} \frac{\partial}{\partial r}\right) k_{i j}\left(\frac{\partial}{\partial x_{j}}+\delta_{j 3} G \hat{H}_{R} \frac{\partial}{\partial r}\right) f+\bar{J} \\
& \frac{\partial f}{\partial t}+\frac{\partial}{\partial x_{i}}\left[\left(u_{i}-v(r) \delta_{i 3}\right) f\right]+\frac{\partial}{\partial r}\left(\frac{c}{r} w f\right) \\
& =\left(\frac{\partial}{\partial x_{i}}+\delta_{i 3} G \hat{H}_{L} \frac{\partial}{\partial r}\right) k_{i j}\left(\frac{\partial}{\partial x_{j}}+\delta_{j 3} G \hat{H}_{R} \frac{\partial}{\partial r}\right) f+\bar{J} .
\end{aligned}
$$

Equation (4.3a) does not use the condition $S \sim S_{q}$, while (4.3b) assumes that $S \sim S_{q}$. The assumption of equilibrium supersaturation might be suitable for mesoscale models with appropriate parameterization of subgrid $\bar{w}_{\text {ef }}$. The assumption of equilibrium supersaturation is not suitable for LES models.

For low-frequency turbulence $\hat{H}_{L, R}=1$, whereas for high-frequency turbulence $\hat{H}_{L, R}=0$. The high-frequency regime, for which generally $S \neq S_{q}$, is thus represented by

$$
\begin{aligned}
\frac{\partial f}{\partial t} & +\frac{\partial}{\partial x_{i}}\left[\left(u_{i}-v(r) \delta_{i 3}\right) f\right]+\frac{\partial}{\partial r}\left(\frac{b S}{r} f\right) \\
& =\frac{\partial}{\partial x_{i}} k_{i j} \frac{\partial}{\partial x_{j}} f+\bar{J} .
\end{aligned}
$$

This type of equation is used in most cloud models with explicit microphysics and describes the broadening of the size spectra due to vertical and horizontal turbulent mixing between the cloud parcels or regions with different properties. The low-frequency regime with the assumption $S \approx S_{q}$, is represented by 


$$
\begin{aligned}
\frac{\partial f}{\partial t} & +\frac{\partial}{\partial x_{i}}\left[\left(u_{i}-v \delta_{i 3}\right) f\right]+\frac{\partial}{\partial r}\left(\frac{c}{r} w f\right) \\
& =\left(\frac{\partial}{\partial x_{i}}+\delta_{i 3} G \frac{\partial}{\partial r}\right) k_{i j}\left(\frac{\partial}{\partial x_{j}}+\delta_{j 3} G \frac{\partial}{\partial r}\right) f+\bar{J}
\end{aligned}
$$

The term $(\partial / \partial r)[(c / r) w f]$ describes "advection" in the space of radii with effective speed $(c / r) w$, inversely proportional to the radius; thus this advection is faster for the smaller droplets and this term alone causes narrowing of the size spectra. Analogous to the term $-k_{i j} \partial f / \partial x_{i}$ which represents the turbulent flux of the droplets in the usual space, the term $-k_{i j} G \partial f / \partial r$ represents the turbulent flux in the space of radii. So the term $k_{r}=G k_{i j}$ is the effective diffusion coefficient in the space of radii, and the parameter $G$ is the scaling factor between the diffusion coefficients in $x_{i}$ and $r$ spaces $\left(G \sim 10^{-9}\right.$ to $\left.10^{-8}\right)$. Since the derivative $-\partial f / \partial r<0$ for the radii less than the modal radius, $r<r_{m}$, the flux of droplets is directed along the gradient toward the smaller radii to the left of the mode, at $r<r_{m}$, and vice versa, $-\partial f / \partial r$ $>0$ at $r>r_{m}$, and the flux is directed to the larger radii. So this "diffusion" in the space of radii tends to smooth the gradients and broaden the size spectrum. The resulting shape of the size spectra is determined by the relative speed of the advection and diffusion in the space of radii and in the usual space. If the effects of advection dominate (e.g., in the vigorous updrafts but with weak turbulence), the spectra may narrow even in the presence of turbulence, which alone might be insufficient to produce the broad spectra.

When written using the left and right operators, the differences are clear between the new equation and the earlier kinetic equations of stochastic condensation reviewed in the introduction. These earlier kinetic equations can be written using our notations after some simplifications as

$$
\begin{aligned}
\frac{\partial f}{\partial t} & +\frac{\partial}{\partial x_{i}}\left[\left(u_{i}-v(r) \delta_{i 3}\right) f\right]+\frac{\partial}{\partial r}\left(\frac{c}{r} w f\right) \\
& =\left(\frac{\partial}{\partial x_{i}}+\delta_{i 3} c \frac{\partial}{\partial r} \frac{1}{r}\right) k_{i j}\left(\frac{\partial}{\partial x_{j}}+\delta_{j 3} c \frac{\partial}{\partial r} \frac{1}{r}\right) f .
\end{aligned}
$$

The new (4.3a) does not assume the quasi-state supersaturation as did earlier kinetic equations. Although (4.3b) assumes this approximation (but with another interpretation of $\bar{w}_{\text {ef }}$ ), it additionally accounts for the nonconservative character of supersaturation and can thus be used in both the low- and high-frequency regimes including the cases when $S \sim S_{q}$, while the previous equations of the type (4.6) are applicable only in the low-frequency regime $\left(\hat{H}_{L, R}=1\right)$ with $S=S_{q}$. Additionally, by virtue of using the microscopic supersaturation fluctuation (2.17) instead of (2.14), the operator $G(\partial / \partial r)$ appears now in $(4.3 \mathrm{a}, \mathrm{b})$ and leads to the solutions of gamma distribution type (see Part II) while the operator $c(\partial / \partial r)(1 / r)$ appears in (4.6) and leads to Gaussian solutions.

In most cloud models with explicit microphysics, the high-frequency form of the kinetic equation (4.4) is used and only a few attempts have been made to use the more complete kinetic equations in the low-frequency approximation (e.g., Vasilyeva et al. 1984). Incorporation into cloud models of the more complete kinetic equations of stochastic condensation in the forms (4.3), (4.5), or (4.6) requires development of efficient economical numerical algorithms for solutions of equations with cross-derivatives by coordinates and radii and the small characteristic time- and spatial scales that determine the condensation process.

\section{Summary}

A new look at the kinetic equations of stochastic condensation has been motivated by the increasing use of explicit microphysics in cloud models and the gap that presently exists between the theory of stochastic condensation and the practical needs of modeling. On one hand, stochastic theory produced several versions of the kinetic equations, which are rather general but perhaps still with some deficiencies as their solutions were of the Gaussian form, while a gamma distribution proves a better representation of observations of drop size spectra. On the other hand, because of the complexity of numerically solving the stochastic kinetic equations, most cloud models use a version of the kinetic equations that is incomplete. Toward addressing these issues, we have derived a new kinetic equation for stochastic condensation that is more suitable for the numerical models and with some simplifications has an analytical solutions of the gamma distribution type.

Relative to the earlier versions of kinetic equation of stochastic condensation, we introduce two new features: 1) consideration of supersaturation as a nonconservative substance with differentiation between the macroscale and microscale supersaturation, and 2) consideration of the supersaturation fluctuations of various frequencies over the entire turbulent spectrum without the assumption of proportionality of supersaturation and vertical velocity. We consider supersaturation as a stochastic field of a nonconservative scalar and relate it to the velocity field by means of spectral expansions in Fourier-Stieltjes integrals. Supersaturation fluctuations $S^{\prime}$ are related to the vertical velocity fluctuations $w^{\prime}$ using the spectral analog of the supersaturation equation; thus $S^{\prime}$ are not proportional to $w^{\prime}$, breaking the link between the $S^{\prime}$ and $w^{\prime}$ and the reversibility of the condensation process typical of the some previous stochastic theories and the parcel models.

Following Srivastava (1989), we distinguish between supersaturation on the microscale and the macroscale. The supersaturation on the macroscale (i.e., generated by the mean vertical velocities and radiation) is typically treated as resulting in the Maxwellian diffusion growth 
of droplets. We showed that supersaturation variations on the microscale give rise to a droplet growth rate that is independent of drop radius. This regime is valid only for high-frequency fluctuations; the dependence of the effective microscale supersaturation on radius weakens with time as the supersaturation relaxes, and the growth rate transforms gradually into the Maxwellian diffusional law for low-frequency fluctuations.

The kinetic equation derived in this paper can be recommended for use in numerical cloud models with explicit microphysics. To apply this equation beyond the high-frequency regime will require economical numerical algorithms for parabolic differential equations with cross derivatives by space and droplet radius. The splitting method can be effectively used for these purposes (Marchuk 1974; Marchuk et al. 1986), which allows reduction during each time step of the multidimensional problem to the sequence of the one-dimensional problems (substeps), and condensation growth can be calculated at the final substep as a zero-dimensional problem by evaluating the integrals of supersaturation over the time step.

The exact and approximate asymptotic analytical solutions to this kinetic equation of the gamma-distribution type are obtained and analyzed in Part II of this paper. These analytical solutions can provide an appropriate basis for parameterization of the cloud processes in the bulk cloud models and the large-scale models.

Acknowledgments. This research was supported by the Department of Energy Atmospheric Radiation Measurement Program and the NASA FIRE program. The assistance of Jody Norman, Olga Melnik, and Alexander Kats in preparation of the manuscript. Oleg Melnik and Dmitry Khvorostyanov are thanked for their help in calculations. Comments on the manuscript from Branko Kosovic and Geoffrey Considine are also appreciated.

\section{APPENDIX}

\section{List of Symbols}

A Coefficient in supersaturation equation, (2.7)

$B_{i j}\left(t-t^{\prime}\right)$ Velocity correlation function

$B_{i j}^{n}\left(t-t^{\prime}\right)$ Nonconservative correlation function of velocity and supersaturation

$B_{i j}^{n n}\left(t-t^{\prime}\right)$ Nonconservative autocorrelation function of supersaturation

$D \quad$ Water vapor diffusion coefficient

c Coefficient of regular condensation, (4.1a)

$c_{p} \quad$ Specific heat capacity

$F_{i j} \quad$ Spectral function of turbulence

$f \quad$ Droplet size distribution function

$G \quad$ Parameter defined in (3.13)

$\hat{H}_{L}, \hat{H}_{R} \quad$ Left and right operators in (4.2)

$J \quad$ Source term on the rhs of kinetic equation

$k_{i j}, k_{z} \quad$ Components of turbulence coefficient $k_{i j}^{n}\left(\tau_{f}\right) \quad$ Nonconservative turbulence coefficient of velocity and supersaturation

$k_{i j}^{n n}\left(\tau_{f}\right) \quad$ Nonconservative turbulence coefficient of supersaturation

$L \quad$ Latent heat of condensation

$l_{j}^{\prime} \quad$ Prandtl's mixing length

$l_{r}^{\prime} \quad$ Mixing length in space of radii

$m \quad$ Mass of a droplet

$N \quad$ Droplet concentration

$p \quad$ Indices of gamma distribution

$Q_{\text {rad }} \quad$ Radiative temperature change

$q, q_{s} \quad$ Specific humidity, saturation specific humidity

$q_{L} \quad$ Liquid water content

$R_{v} \quad$ Gas constant of water vapor

$r, \bar{r} \quad$ Droplet radius, mean radius

$r_{\text {ef }} \quad$ Effective optical radius

$\dot{r} \quad$ Droplet growth rate

$\dot{r}^{\prime} \quad$ Fluctuations in growth rate

$S \quad$ Supersaturation

$S^{\prime} \quad$ Supersaturation fluctuations

$S_{\tilde{S}_{q}} \quad$ Quasi-steady-state supersaturation, (2.9)

$\tilde{S}_{q}^{q} \quad$ Relative quasi-steady-state supersaturation, (2.9a)

$\begin{array}{ll}t & \text { Time } \\ T & \text { Temperature }\end{array}$

$U, u_{i} \quad$ Vector and components of wind speed

$v(r) \quad$ Terminal velocity

$w \quad$ Vertical velocity

$w_{\text {rad }} \quad$ Radiative-effective velocity, (2.8)

$w_{\text {ef }} \quad$ Effective velocity

$x, y, z \quad$ Coordinates

$\gamma_{d}, \gamma_{s} \quad$ Dry and moist adiabatic lapse rates

$\hat{\Delta} \quad$ Operator of turbulent diffusion

$\Gamma \quad$ Psychrometric correction in equation for growth rate

$\delta(x) \quad$ Dirac's delta function

$\delta_{i j} \quad$ Kronecker's symbol

$\varepsilon_{c} \quad$ Condensation rate

$\rho_{v}, \rho_{w}, \rho_{a}$ Densities of vapor, water, and air

$\sigma_{r} \quad$ Relative spectral dispersions

$\theta(x) \quad$ Heaviside step function

$\tau_{f} \quad$ Supersaturation relaxation (absorption) time

$\tau_{L} \quad$ Lagrangian turbulent time

$\omega \quad$ Turbulent frequency

$\omega_{p} \quad$ Frequency of supersaturation relaxation

\section{REFERENCES}

Austin, P. H., M. B. Baker, A. M. Blyth, and J. B. Jensen, 1985: Small-scale variability in warm continental cumulus clouds. $J$. Atmos. Sci., 42, 1123-1138.

Baker, M. B., and J. Latham, 1979: The evolution of the droplet spectra and the rate of production of embryonic raindrops in small cumulus clouds. J. Atmos. Sci., 36, 1612-1615.

-, R. E. Briedenthal, T. W. Choularton, and J. Latham, 1984: The effects of turbulent mixing in clouds. J. Atmos. Sci., 41, 299304.

Broadwell, J. E., and R. E. Briedenthal, 1982: A simple model of 
mixing and chemical reaction in a turbulent shear layer. J. Fluid. Mech., 125, 397-410.

Browning, K. A., Ed., 1994: GEWEX Cloud System Study (GCSS). Science Plan. IGPO Publishing Series, No. 11, 62 pp. [Available from WCRP, 7 av. Paix, CH1211 Geneva 2, Switzerland.]

Buikov, M. V., 1961: Kinetics of distillation in a polidisperse fog. Izv. Acad. Sci. USSR, Ser. Geophys., 7, 1058-1065.

_ 1963: A method of the kinetic equations in the theory of clouds. Proc. All-Union Meteorol. Conf., Leningrad, USSR, Vol. 5, 122 128.

—_, and A. M. Pirnach, 1973: A numerical model of a two-phase stratiform cloud with explicit account for the microstructure. Izv. Acad. Sci. USSR, Atmos. Oceanic Phys., 9, 486-499.

— diative fog and stratus formation taking into account interaction among dynamic, radiative and microphysical processes. Proc. Sixth Int. Conf. on Cloud Phys., Boulder, CO, Amer. Meteor. Soc., 392-395.

Clark, T. L., 1973: Numerical modelling of the dynamics and microphysics of warm cumulus convection. J. Atmos. Sci., 30, 857878.

Considine, G., and J. A. Curry, 1996: A statistical model of drop size spectra for stratocumulus clouds. Quart. J. Roy. Meteor. Soc., 122, 611-634.

Cooper, W. A., 1989: Effect of variable droplet growth histories on droplet size distributions. Part I: Theory. J. Atmos. Sci., 46, 1301-1311.

Cotton, W. R., and R. A. Anthes, 1989: Storm and Cloud Dynamics. Academic Press, 883 pp.

Curry, J. A., 1986: Interactions among turbulence, radiation and microphysics in Arctic stratus clouds. J. Atmos. Sci., 43, 525-538.

— - E. E. Ebert, and G. F. Herman, 1988: Mean and turbulent structure of the summertime Arctic cloudy boundary layer. Quart. J. Roy. Meteor. Soc., 114, 715-746.

Feingold, G., B. Stevens, W. R. Cotton, and R. L. Walko, 1994: An explicit cloud microphysics/LES model designed to simulate the Twomey effect. Atmos. Res., 33, 207-233.

Flossman, A. I., W. D. Hall, and H. P. Pruppacher, 1985: A theoretical study of the wet removal of atmospheric pollutants. Part I: The redistribution of aerosol particles captured through nucleation and impaction scavenging by growing cloud droplets. J. Atmos. Sci., 42, 583-606.

Fukuta, N., and L. A. Walter, 1970: Kinetics of hydrometeors growth from a vapor-spherical model. J. Atmos. Sci., 27, 1160-1172.

Kabanov, A. S., I. P. Mazin, and V. I. Smirnov, 1970: Effect of spatial inhomogeneity of nucleating droplets on their size spectrum in a cloud. Izv. Acad. Sci. USSR, Atmos. Oceanic Phys., 6, 265277

Khvorostyanov, V. I., 1982: A two-dimensional time-dependent microphysical model of advective-radiative fog and low clouds. Sov. Meteor. Hydrol., 7, 16-28.

_ 1995: Mesoscale processes of cloud formation, cloud-radiation interaction and their modelling with explicit cloud microphysics. Atmos. Res., 39, 1-67.

_ , and K. Sassen, 1998: Cirrus cloud simulation using explicit microphysics and radiation. Part I: Model description. J. Atmos. Sci., 55, 1808-1821.

- , and J. A. Curry, 1999a: Toward the theory of stochastic condensation in clouds. Part II: Analytical solutions of the gamma distribution type. J. Atmos. Sci., 56, 3997-4013.

$\longrightarrow$, and - 1999b. A simple analytical model of aerosol properties with account for hygroscopic growth. Part I: Equilibrium size spectra and CCN activity spectra. J. Geophys. Res., 104 (D2), 2163-2174.

Kogan, Y. L., 1991: The simulation of a convective cloud in a 3D model with explicit microphysics. Part I: Model description and sensitivity experiments. J. Atmos. Sci., 48, 1160-1189.

Latham, J., and R. L. Reed, 1977: Laboratory studies of the effects of mixing on the evolution of cloud droplet spectra. Quart. J. Roy. Meteor. Soc., 103, 297-306.

Levin, L. M., and Y. S. Sedunov, 1966: Stochastic condensation of drops and kinetics of cloud spectrum formation. J. Rech. Atmos., 2, 425-432.

Manton, M. J., 1979: On the broadening of a droplet distribution by turbulence near cloud base. Quart. J. Roy. Meteor. Soc., 105, 899-914.

_ 1981: Reply to the comments on the paper "On the broadening of a droplet distribution by turbulence near cloud base." Quart. J. Roy. Meteor. Soc., 112, 977-978.

Marchuk, G. I., 1974: Numerical Methods in Weather Prediction. Academic Press, $321 \mathrm{pp}$.

- , K. Y. Kondratyev, V. V. Kozoderov, and V. I. Khvorostyanov, 1986: Clouds and Climate. Hydrometeorological Publishers, 612 pp.

Mazin, I. P., 1968: The stochastic condensation and its effect on the formation of cloud drop size distribution. Proc. Int. Conf. Cloud Phys., Toronto, ON, Canada, Amer. Meteor. Soc., 67-71.

Merkulovich, V. M., and A. S. Stepanov, 1977: Hygroscopicity effects and surface tension forces during condensational growth of cloud droplet in the presence of turbulence. Izv. Akad. Sci. USSR, Atmos. Oceanic Phys., 13, 163-171.

— , and — 1981: Comments on the paper "On the broadening of a droplet distribution by turbulence near cloud base" by M. J. Manton (1979). Quart. J. Roy. Meteor. Soc., 112, 976-977.

Monin, A. S., and A. M. Yaglom, 1967: Statistical Hydromechanics. Vol. 2. Nauka Press, 676 pp.

Mordy, W. A., 1959: Computations of the growth by condensation of a population of cloud droplets. Tellus, 11, 16-44.

Neiburger, M., and C. W. Chien, 1960: Computations of the growth of cloud drops by condensation using an electronic digital computer. Geophys. Monogr., No. 5, Amer. Geophys. Union, 191208.

Pruppacher, H. R., and J. D. Klett, 1997: Microphysics of Clouds and Precipitation. 2d ed. Kluwer, 997 pp.

Sassen, K., D. O. Starr, and T. Uttal, 1989: Mesoscale and microscale structure of cirrus clouds: Three case studies. J. Atmos. Sci., 46, 371-396.

Sedunov, Y. S., 1965: The fine structure of the clouds and its role in formation of the cloud droplet spectra. Izv. Acad. Sci. USSR, Atmos. Oceanic. Phys., 1, 722-731.

— 1974: Physics of Drop Formation in the Atmosphere. Wiley, $234 \mathrm{pp}$.

Squires, P., 1952: The growth of cloud drops by condensation. Aust. J. Sci. Res., A5, 59-86.

Srivastava, R. C., 1989: Growth of cloud drops by condensation: A criticism of currently accepted theory and a new approach. $J$. Atmos. Sci., 46, 869-887.

Stepanov, A. S., 1975: Condensational growth of cloud droplets in a turbulized atmosphere. Izv. Akad. Sci. USSR, Atmos. Oceanic Phys., 11, 27-42.

Telford, J. W., and S.K. Chai, 1980: A new aspect of condensation theory. Pure Appl. Geophys., 118, 720-742.

Twomey, S., 1959: The nuclei of natural cloud formation. II. The supersaturation in natural clouds and the variation of cloud droplet concentration. Geophys. Pura Appl., 43, 243-249.

Tzivion, S., G. Feingold, and Z. Levin, 1987: An efficient numerical solution to the stochastic collection equation. J. Atmos. Sci., 44, 3139-3149.

Vasilyeva, K. I., V. M. Merkulovich, and A. S. Stepanov, 1984: Behavior of the droplet spectrum in a turbulized two-phase cloud. Sov. Meteor. Hydrol., 11, 31-42.

Voloshchuk, V. M., and Y. S. Sedunov, 1977: A kinetic equation for the evolution of the droplet spectrum in a turbulent medium at the condensation stage of cloud development. Sov. Meteor. Hydrol., 3, 3-14.

Warner, J., 1969: The microstructure of cumulus cloud. Part I: General features of the droplet spectrum. J. Atmos. Sci., 26, 1049-1059. 